Article

\title{
Evaluate on the Decoupling of Tourism Economic Development and Ecological-Environmental Stress in China
}

\author{
Xiaohua Qin ${ }^{1,2}$ and Xingming $\mathrm{Li}^{1, *}$ \\ 1 Key Laboratory for Geographical Process Analysis \& Simulation Hubei Province, School of City and \\ Environment, Central China Normal University, Wuhan 430079, China; niq488@sina.com \\ 2 School of Tourism and Culture, Nanning Normal University, Nanning 530001, China \\ * Correspondence: xingming@mail.ccnu.edu.cn; Tel.: +86-180-7105-9360
}

check for updates

Citation: Qin, X.; Li, X. Evaluate on the Decoupling of Tourism Economic Development and

Ecological-Environmental Stress in China. Sustainability 2021, 13, 2149.

https://doi.org/10.3390/su13042149

Academic Editor: Andrea Cirà

Received: 25 January 2021

Accepted: 11 February 2021

Published: 17 February 2021

Publisher's Note: MDPI stays neutral with regard to jurisdictional claims in published maps and institutional affiliations.

Copyright: (C) 2021 by the authors Licensee MDPI, Basel, Switzerland. This article is an open access article distributed under the terms and conditions of the Creative Commons Attribution (CC BY) license (https:// creativecommons.org/licenses/by/ $4.0 /)$.
Abstract: Tourism economic development is increasingly dependent on resources and environment. Exploring the relationship between tourism economic development and ecological-environmental (eco-environmental) stress is of great significance to promote the high-quality growth of tourism and the sustainable and coordinated development of ecological environment. By constructing a tourism economic development index and an eco-environmental stress index, this study analyzes the temporal and spatial evolution of tourism economic development and eco-environmental stress from 2009 to 2018 in China. It uses a decoupling model to evaluate the relationship between tourism economic development and ecological-environmental stress, and analyzes the reasons for the changes of decoupling relationship. The results show that: (1) During the study period, the development of tourism economy and the eco-environmental stress present a certain time-space effect characteristics. The stress index of China's tourism economic development and ecological environment showed a fluctuating trend of first decreasing and then increasing, with obvious spatial hierarchical differences and weak agglomeration characteristics, and prominent regional imbalances. The tourism economic development level in the eastern region was higher than that in the central and western regions, while the ecological environment stress in the central region was greater than that in the eastern and western regions. (2) The relationship between tourism economic development and ecological environmental stress of China's provinces has experienced eight states: Expansive negative decoupling, strong negative decoupling, weak negative decoupling, recessive coupling, expansive coupling, strong decoupling, weak decoupling, and recessive decoupling. During the study period, the state of optimal strong decoupling tends to weaken. Under the constraints of local policy orientation and regional economic development level, the overall decoupling optimization could not be achieved spatially. The decoupling state was always in an unsustainable non-optimal stage. (3) The reasons for the differential changes in the decoupling index between tourism economic development and ecological environmental stress in Chinese provinces come from investment-driven, resource-driven, innovation-driven, and environmental compliance push. This study can provide practical reference for promoting the high-quality development of tourism and the sustainable development of ecological environment.

Keywords: tourism economy; eco-environmental stress; decoupling evaluation; spatio-temporal difference

\section{Introduction}

In recent years, China's tourism economy has entered a stage of rapid growth, with increasing demand for tourism. The process of tourism economic growth is the process of sustainable development and utilization of resources, and at the same time, it will continue to discharge waste to the environment. The dependence of tourism economic development on resources and environment is increasing [1]. Tourism economic activity inevitably certain stress and the influence on the ecological environment [2]. The process of tourism 
economic development is usually to focus on the short-term economic interests and to ignore the sustainable healthy development of resources and environment. The extensive economic growth mode is especially serious beyond the tourist ecological carrying capacity as it causes irreversible damage to the ecological environment. Therefore, exploring the tourism economic development and ecological environment sustainable development has important practical significance. In the report of the 19th National Congress of the Communist Party of China, the concept of ecological civilizationis proposed.It should be fully integrated into economic construction, sticking to the fact that green water and green mountains are valuable, and fully promoting green development. With the emergence of a series of problems such as air pollution, water pollution, resource depletion, and deterioration of the ecological environment, the relationship between economic growth and ecological-environmental stress has gradually attracted widespread attention. Tourism is the world's largest service sector, and it continues to grow [3]. The sustainable development of the tourism economy, an important strategic pillar industry of the national economy, plays a role in boosting the sustainability of the entire national economy. However, the intrinsic requirement of sustainable development of the tourism economy is that tourism activities do not destroy the existing environment of the tourist destination, and the existing tourism resources should be protected and rationally-utilized without damaging the potential tourism resources, to ensure the harmonious development of the tourism economy and the natural environment. Under the background of the development strategy of ecological civilization in the new era, research is needed in how to optimize resource allocation and improve utilization efficiency in accordance with the inherent requirements of sustainable economic development, how to guide high-quality development of tourism economy, and how to realize tourism economic growth without sacrificing the ecological environment. These have become an urgent problems to be solved. Based on the realistic requirements of harmonious coexistence between humans and nature, correctly handling the relationship between tourism economic development and ecological environment is of great significance to promote the sustainable, healthy, and high-quality development of tourism.

At present, some achievements have been made in the study of the relationship between tourism and ecological environment. Foreign studies on the relationship between tourism and ecological environment mainly focus on the impact of tourism activities on specific ecological factors [4,5], tourism environmental capacity [6], tourism environmental carrying capacity [7], and tourism ecological security [8]. Domestic studies on the relationship between tourism and ecological environment mainly focus on the coupling mechanism of tourism and ecological environment $[9,10]$, the coordination of tourism resource development and ecological environment [11], tourism ecological security [12], and tourism environmental carrying capacity evaluation [13]. In addition, domestic and foreign scholars have also discussed the relationship between tourism and ecological environment from the perspective of sustainable tourism [14-17]. Sustainable tourism emphasizes that ecological environment protection should be paid attention to in the process of developing tourism, and avoiding the destruction of ecological environment caused by the excessive development of tourism resources. The content of the research on the relationship between tourism and the ecological environment is becoming increasingly abundant, and the research methods are gradually diversified, especially when the coupling coordination model and the stress-state-response (PSR) model are most widely used. The term "decoupling" was first applied in the field of physics, which refers to the process in which the interaction relationship between two or more physical quantities no longer exists and is separated from each other [18]. In 1966, Carter first applied the concept of "decoupling" to explain the relationship between economic growth and energy consumption [19]. In 2002, the Organization for Economic Cooperation and Development (OECD) first proposed the "decoupling theory" to explore how to block the relationship between economic growth and environmental impact [20]. At present, decoupling theory has become an effective analysis method to measure the relationship between economic development, resource 
consumption and environmental stress, and has been widely used abroad. At first, OECD used decoupling theory to comprehensively explain the decoupling relationship between economic growth and environmental stress in 30 member states. And then it combined the theory to analyze the equilibrium relationship in the fields of agriculture [21], energy [22], transportation [23], environment [24], atmospheric emissions [25], etc. The application of decoupling model in the study of the relationship between economic development and resources and environment has gradually become a hot topic in academic circles.In recent years, domestic scholars have gradually applied decoupling theory to the study of the relationship between economic development and resources environment [26], environmental pollution [27], energy consumption [28], arable land [29], water resources utilization [30], urbanization [31], construction land [32], carbon emissions [33], etc. In summary, the decoupling method has not been used to systematically discuss the relationship between tourism economic development and ecological environmental stress in the existing studies.

Although the research on the relationship between tourism and the ecological environment and the theory of decoupling has made great progress, there are still limitations. Firstly, the research object focus on agriculture, the environment pollution, energy, land use, urbanization of decoupling analysis. Using decoupling theory systemic evaluation of China's tourism economy development and ecological environment stress has not been found. It is not conducive to the sustainable healthy development of tourism. Then, the sustainability of the tourism industry and its promotion of the ecological environment is difficult to measure. Secondly, the research paradigm is mainly focused on the interpretation of mathematical models and empirical research, mainly analyzed from the perspectives of statistics, economics, and management. It lacks research from the perspective of geography, and the degree of decoupling between tourism economic development and ecological environmental stress from the perspective of space has not been found. Moreover, compared with other methods, decoupling analysis methods focus on deduction from the time series analysis method, can travel through simple quantitative relation to comprehensively reflect the internal law of economic development and ecological environment stress. It can use panel data in horizontal comparison, accurately identify the tourism economic development and ecological environmental stress in a certain stage of development of dynamic information, and can predict development trends.

Therefore, based on the existing relevant results, and relevant theories of geography and economics, this study aims to evaluate the relationship between tourism economic development and ecological environmental stress in China from 2009 to 2018 from the perspective of time and space by using decoupling model. First, the evaluation index system of tourism economic development and ecological environmental stress is constructed, and the entropy weight method is used to measure the development level of China's tourism economy and the status of ecological environmental stress. The characteristics of China's tourism economic development and the degree of ecological environmental stress are clarified. Next, considering the criteria of decoupling, this paper analyzes the degree of decoupling between China's tourism economic development and ecological environmental stress. Finally, the reasons for the change in the decoupling relationship are explained based on the results of decoupling evaluation. The research results can provide a theoretical understanding of the spatio-temporal evolution of China's tourism economic development, offer practical reference for the sustainable and healthy development of the ecological environment and the high-quality development of tourism. They also have certain reference value for the sustainable development of tourism in other countries.

\section{Research Methods and Data Sources}

\subsection{Evaluation Index System Construction}

Constructing an evaluation index system is an important basis for studying the interaction between tourism economic development and ecological environmental stress in China. Tourism economic development is a complex systematic project. The selection of indicators should be based on the stage characteristics of regional economy, and based on the 
principles of the times, systematization, scientificity, rationality, and data availability of the indicators, the connotation of tourism industry is specified and indexed. This study focuses on input-output theory based on the understanding of the connotation requirements of high-quality tourism development in the new era, and on the basis of fully referring to the experience of Fang et al. [34], Guo et al. [35], and Zhou et al. [36] in constructing indicators. From the four dimensions of tourism enterprise scale, tourism capital investment, tourism reception level and tourism industry performance, this paper constructs an evaluation index system of tourism economic development level, which is composed of four primary indicators and corresponding 10 secondary indicators (Table 1).Tourism economic development and ecological environment are closely related, and the ecological environment stress index is constructed to comprehensively reflect the ecological environment. To measure the stress of an ecological environment, we should not only consider the consumption of ecological resources and energy at the input end, but also consider the material emissions and measure the ecological environment load through the threat degree of natural disasters. Therefore, this study combines the principle of circular and cumulative causation, which is in line with the connotation requirements of sustainable development. Based on the existing research results of $\mathrm{Xu}$ et al. [37], Zhu et al. [38], and Du et al. [39], 14 indicators were selected to characterize the eco-environmental stress from the three dimensions of resource consumption conditions, environmental pollution status, and natural threat levels, respectively. The specific evaluation system is shown in Table 1.

Table 1. Evaluation index system of tourism economic development and ecological environmental stress.

\begin{tabular}{|c|c|c|c|}
\hline System & Primary Indicators & Secondary Indicators & Unit \\
\hline \multirow{10}{*}{$\begin{array}{l}\text { Tourism economic } \\
\text { development system }\end{array}$} & \multirow{3}{*}{ Tourism enterprise scale } & Number of tourist attractions & individual \\
\hline & & Number of star-rated hotels & individual \\
\hline & & Number of travel agencies & individual \\
\hline & \multirow{2}{*}{ Tourism capital investment } & Total tourism investment in & Ten thousand yuan \\
\hline & & $\begin{array}{l}\text { fixed assets } \\
\text { Number of tourism employees }\end{array}$ & People \\
\hline & \multirow{3}{*}{ Tourism recception level } & Number of domestic tourists & Million people \\
\hline & & Number of inbound tourists & Million people \\
\hline & & Domestic tourism revenue & Billion yuan \\
\hline & \multirow[t]{2}{*}{ Tourism industry performance } & $\begin{array}{l}\text { Foreign exchange earnings from } \\
\text { international tourism }\end{array}$ & Million dollars \\
\hline & & $\begin{array}{l}\text { The proportion of total tourism } \\
\text { revenue in the tertiary industry }\end{array}$ & $\%$ \\
\hline \multirow{14}{*}{$\begin{array}{l}\text { Ecological environmental } \\
\text { stress system }\end{array}$} & \multirow{5}{*}{$\begin{array}{l}\text { Resource consumption } \\
\text { conditions }\end{array}$} & Electricity consumption & Billion kilowatt hours \\
\hline & & Water consumption & Billion cubic meters \\
\hline & & Total energy consumption & Million tons of standard coal \\
\hline & & Green space area & Million hectares \\
\hline & & Urban construction land area & Square kilometers \\
\hline & \multirow{5}{*}{$\begin{array}{l}\text { Environmental pollution } \\
\text { status }\end{array}$} & Sulfur dioxide emissions & Million tons \\
\hline & & \multirow{4}{*}{$\begin{array}{l}\text { Smoke (dust) emissions } \\
\text { Total amount of wastewater } \\
\text { discharge } \\
\text { Industrial solid waste generation } \\
\text { Harmless disposal capacity of } \\
\text { domestic waste }\end{array}$} & Ton \\
\hline & & & Million tons \\
\hline & & & Million tons \\
\hline & & & Million tons \\
\hline & \multirow{4}{*}{ Natural threat level } & \multirow{4}{*}{$\begin{array}{c}\text { Amount of pesticides used } \\
\text { Area affected by natural disasters } \\
\text { Number of people affected by } \\
\text { natural disasters } \\
\text { Number of occurrences of } \\
\text { geological disasters }\end{array}$} & Million tons \\
\hline & & & Thousands of hectares \\
\hline & & & Million people \\
\hline & & & Times \\
\hline
\end{tabular}




\subsection{Evaluation Method}

\subsubsection{Entropy Weight Method}

This study adopts the entropy weight method to comprehensively measure the level of tourism economic development and ecological environment stress. To eliminate the dimensional differences of evaluation indexes and make them more comparable, the range method was used to standardize the dimensional data [40], among which, different processing methods were chosen for positive and negative indicators, and for positive indicators: $Z_{\mathrm{ij}}^{\prime}=\left(Z_{\mathrm{ij}}-Z_{\mathrm{ijmin}}\right) /\left(Z_{\mathrm{ijmax}}-Z_{\mathrm{ijmin}}\right)$; for negative indicators: $Z_{\mathrm{ij}}^{\prime}=$ $\left(Z_{\mathrm{ijmax}}-\mathrm{Z}_{\mathrm{ij}}\right) /\left(\mathrm{Z}_{\mathrm{ijmax}}-\mathrm{Z}_{\mathrm{ijmin}}\right)$. After standardization, the weight values of the indicators were determined, and finally the linear weighting model was used to solve the tourism economic development index and eco-environmental stress index of China's provinces from 2009 to 2018.The higher value of tourism economic development index reflects the higher level and quality of tourism economic development, and vice versa; the higher value of eco-environmental stress index indicates the greater stress on the ecological environment, and vice versa. Among them, the calculation formula of each index weight value is as follows:

Implementation of weight conversion:

$$
S_{i j}=\frac{Z_{i j}^{\prime}}{\sum_{i=1}^{n} Z_{i j}^{\prime}}
$$

Calculating entropy value:

$$
P_{j}=-\frac{1}{\ln n} \sum_{i=1}^{m} S_{i j} \ln S_{i j}
$$

Calculating index weight:

$$
A_{j}=\frac{1-P_{j}}{\sum_{i=1}^{m} P_{j}}
$$

The formula for calculating tourism economic development index and eco-environmental stress index is as follows:

$$
\begin{aligned}
& \text { TEDI }=\sum_{i=1}^{n} T_{i} A_{i} \\
& \text { EEPI }=\sum_{j=1}^{n} T_{j} A_{j}
\end{aligned}
$$

where: TEDI and EEPI are tourism economic development index and eco-environmental stress index, respectively; $Z_{i}$ and $Z_{j}$ are the standardized values of various indicators in the tourism economic development system and ecological environmental stress system, respectively; $A_{i}$ and $A_{j}$ are the weight values corresponding to $T_{i}$ and $T_{j}$, respectively.

\subsubsection{Decoupling Evaluation Model}

The purpose of this study is to explore the relationship between tourism economic development and ecological environmental stress, and the decoupling evaluation model can be used to determine the decoupling state of the two. According to the relevant research results at home and abroad [41-43], with full reference to Tapio decoupling elasticity coefficient method [23], this paper constructs the decoupling evaluation model of tourism economic development and ecological environment stress, according to the following expression:

$$
\mathrm{DI}_{\mathrm{t}}=\frac{\Delta \mathrm{EEPI}_{\mathrm{t}}}{\Delta \mathrm{TEDI}_{\mathrm{t}}}=\frac{\left(\mathrm{EEPI}_{\mathrm{c}}-\mathrm{EEPI}_{\mathrm{b}}\right) / \mathrm{EEPI}_{\mathrm{b}}}{\left(\mathrm{TEDI}_{\mathrm{c}}-\mathrm{TEDI}_{\mathrm{b}}\right) / \mathrm{TEDI}_{\mathrm{b}}}
$$


where: $\mathrm{DI}_{t}$ represents the decoupling index of eco-environmental stress on tourism economic development in period $\mathrm{t} ; \Delta \mathrm{EEPI} \mathrm{I}_{\mathrm{t}}$ represents the rate of change of eco-environmental stress index in period $t, \triangle T_{E D I}$ represents the rate of change of tourism economic development index in period $\mathrm{t}$; $\mathrm{EEPI}_{\mathrm{c}}$ and $\mathrm{EEPI}_{\mathrm{b}}$ represent the eco-environmental stress index in the end and beginning years of period $t$, respectively; $\mathrm{TEDI}_{\mathrm{c}}$ and $\mathrm{TEDI}_{\mathrm{b}}$ represent the tourism economic development index in the end and beginning years of period $t$, respectively.

Combined with the range of values of the decoupling index DI, "decoupling" is divided into three categories: Decoupling, coupling, and negative decoupling. It is also divided into eight subcategories: Expansive negative decoupling, strong negative decoupling, weak negative decoupling, recessive coupling, expansive coupling, strong decoupling, weak decoupling, and recessive decoupling [44], among which the strong decoupling type belongs to the optimal development state. The specific determination criteria are shown in Figure 1.

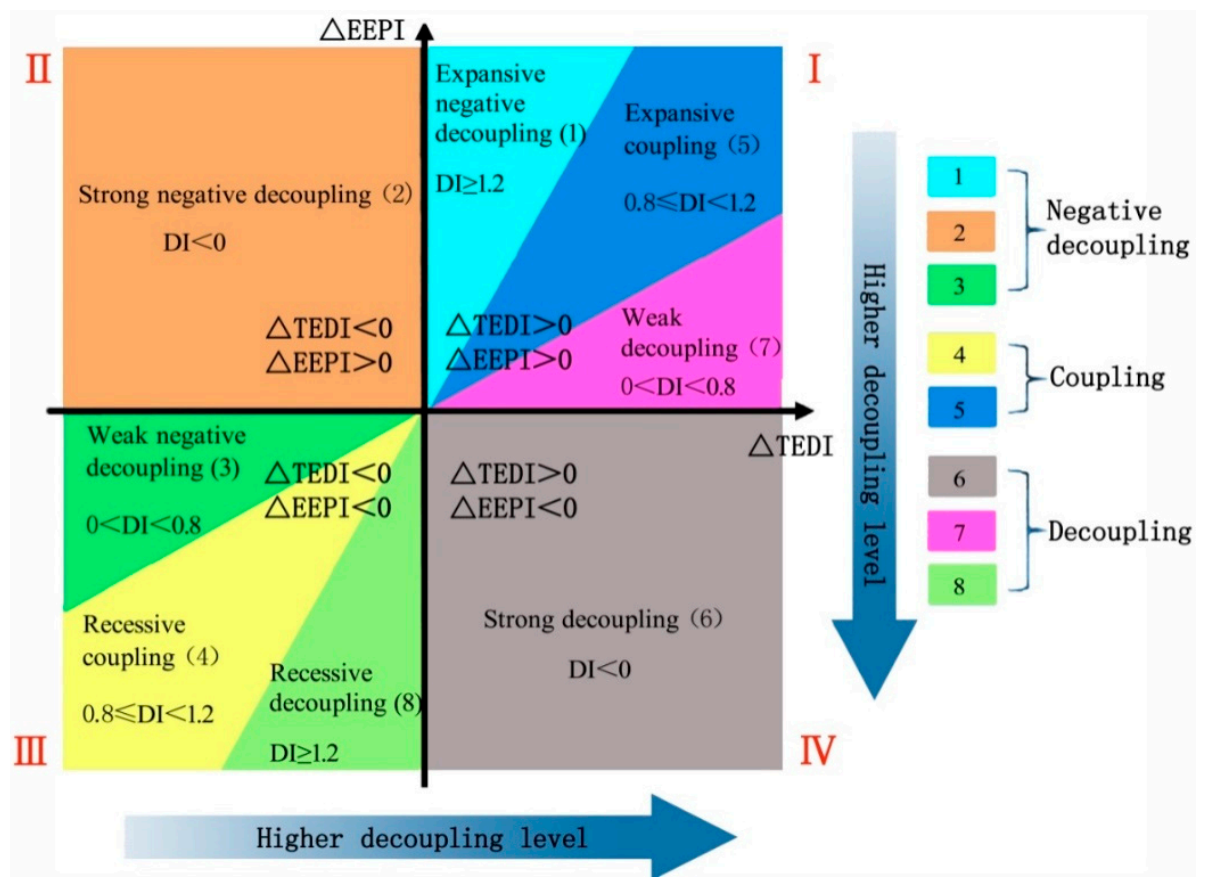

Figure 1. Criteria for determining the decoupling index between tourism economic development and ecological environmental stress.

\subsubsection{Exploratory Spatial Data Analysis Method}

Exploratory spatial data analysis method is used to determine whether there is a correlation between the observation values of a certain position and its adjacent position in space, and to explore its spatial agglomeration characteristics [45]. This study uses the global Moran's I index to measure the spatial correlation and degree of agglomeration of the decoupling state of China's tourism economic development and the ecological environmental stress. Usually, Moran's I index value is in the range of $[-1,1]$. When Moran's I value is less than 1 and close to -1 , it indicates that the decoupling state is in negative spatial correlation. When Moran's I value is equal to 0 , it indicates that the decoupling state has no spatial correlation and is in a random distribution state. When Moran's I value is greater than 0 , it indicates that the decoupling state is spatially positively correlated and in an agglomerative distribution state, and the closer to 1 , the more significant the degree of agglomeration. The calculation formula is [46]:

$$
\text { Moran' } \mathrm{sI}=\mathrm{n} \frac{\sum_{\mathrm{i}=1}^{\mathrm{n}} \sum_{\mathrm{j}=1}^{\mathrm{n}} \mathrm{w}_{\mathrm{ij}}\left(x_{\mathrm{i}}-\bar{x}\right)\left(x_{\mathrm{j}}-\bar{x}\right)}{\sum_{\mathrm{i}=1}^{\mathrm{n}} \sum_{\mathrm{j}=1}^{\mathrm{n}} \mathrm{w}_{\mathrm{ij}} \sum_{\mathrm{i}=1}^{\mathrm{n}}\left(x_{\mathrm{j}}-\bar{x}\right)^{2}}
$$


where: $x_{\mathrm{i}}$ and $x_{\mathrm{j}}$ represent the decoupling index of the $\mathrm{i}$ and $\mathrm{j}$ provinces, respectively; $\bar{x}$ is the average value of the decoupling index, $\mathrm{w}_{\mathrm{ij}}$ is the space vector matrix; $\mathrm{n}$ is the number of provinces in China.

\subsection{Data Sources}

The data used in this paper were obtained from the "China Tourism Statistical Yearbook" (including copies), "China Statistical Yearbook", "China Environment Statistical Yearbook", "China Energy Statistical Yearbook", and the statistical yearbooks of Chinese provinces, selected for a time span from 2009-2018. A small part of the missing data in individual years is supplemented by interpolation method. In addition, in view of the serious lack of data in Tibet, Hong Kong, Macao, and Taiwan, it was excluded from the research sample. This study uses the basic data of geographic information, mainly derived from the 1:4 million database of the National Geographic Information Center.

\section{Results and Analysis}

\subsection{Characteristics of Changes in the Level of Tourism Economic Development}

Equation (4) was applied in calculating China's tourism economic development level index from 2009 to 2018, as shown in Figure 2, so as to obtain the characteristics of the development and change of tourism economy in China and various regions. From the perspective of time series change trend: China's tourism economic development index first dropped from 0.3059 in 2009 to 0.2991 in 2011, then rose to 0.3048 in 2013, then fell to 0.2731 in 2015, and finally rose again to 0.3249 in 2018, showing an overall fluctuating change trend of first declining and then rising. The tourism economic development index of the eastern, central, and western provinces in China also showed an overall fluctuating trend of first declining and then rising, and there are significant differences among different regions. The tourism economic development index of eastern provinces was higher than the national average, while the tourism economic development index of the central and western regions was lower than the national average. In summary, the tourism economy of the eastern provinces of China has always been at a high level of development, which can play a role in radiating and driving the development of the central and western provinces. However, the development level of the tourism economy of the central and western provinces is relatively low. There is an urgent need to increase the scale of investment in the tourism industry and optimize the development of tourism resources, thereby promoting the high-quality development of the tourism economy.

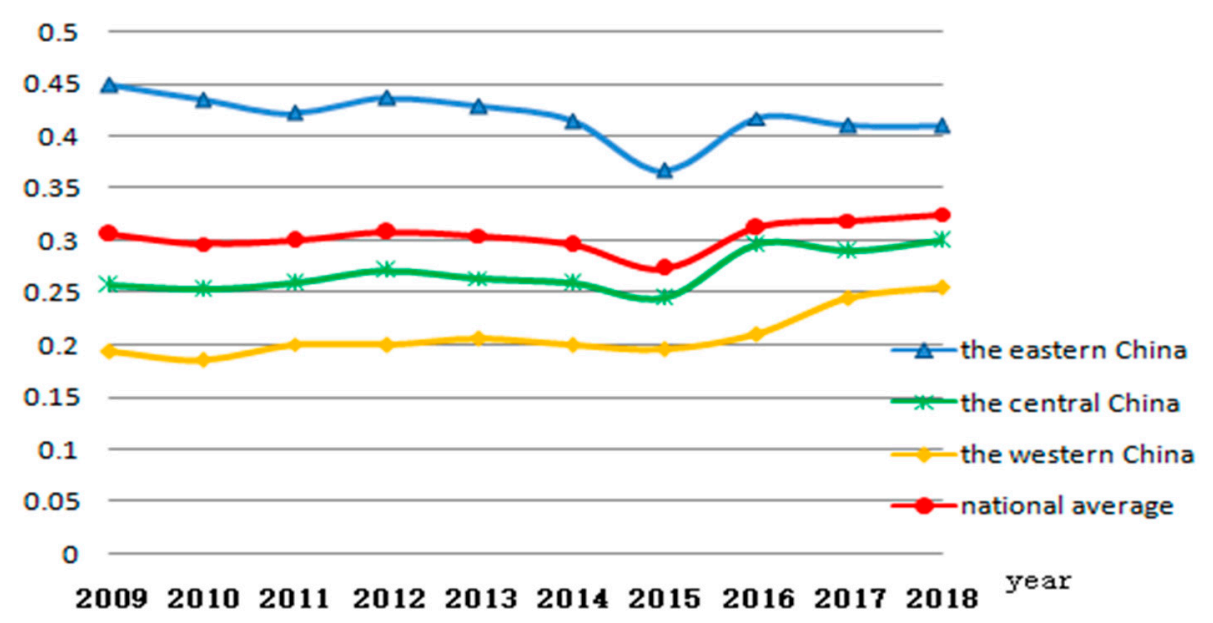

Figure 2. Time series change trend of tourism economic development index from 2009 to 2018 in China.

In order to further explore the spatial difference characteristics of tourism economic development level of China's provinces, this study uses ArcGIS 10.2 software to spatially 
visualize the calculation results of the tourism economy development index. Combined with the Jenks natural breaks method, it is divided into 5 level-areas: Higher-level area, high-level area, medium-level area, low-level area, and lower-level area. It is selected the 3 years in 2009, 2013, and 2018 to carry out the specific analysis, and then drew the spatial evolution trend map of China's tourism economic development index (Figure 3).

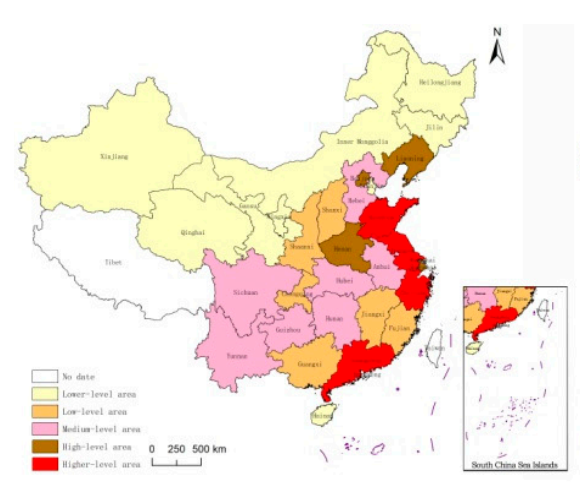

(a) 2009

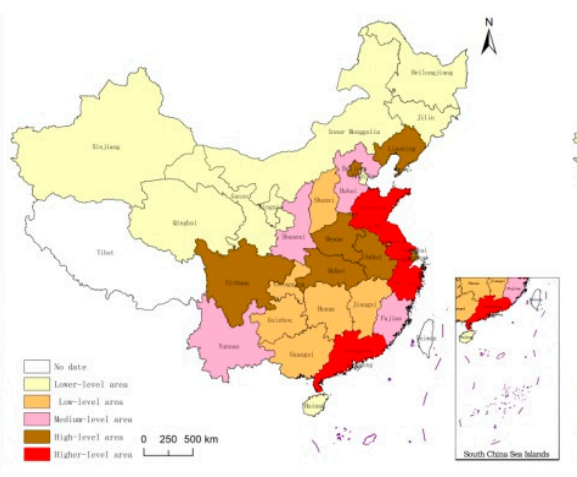

(b) 2013

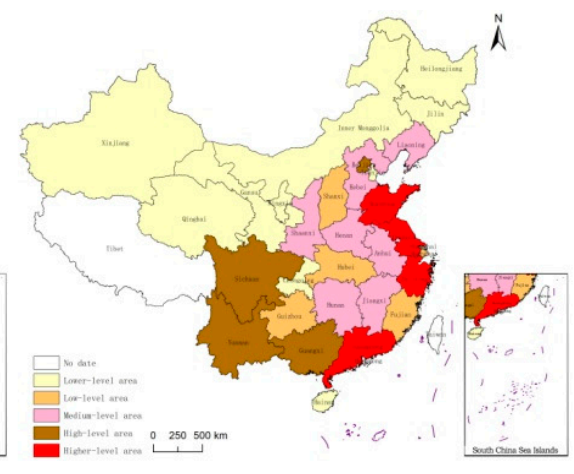

(c) 2018

Figure 3. Spatial pattern oftourism economic development index from 2009 to 2018 in China. (a-c) spatial evolution trend map of China's tourism economic development index.

From the perspective of spatial evolution pattern, the tourism economic development index presents the distribution characteristics of "the lower-level areas are stably concentrated in the northwest, and the higher-level areas are significantly distributed in the eastern coast". In 2009, provinces with higher-level area of tourism economic development were concentrated in eastern developed provinces such as Shandong, Jiangsu, Zhejiang, and Guangdong. Lower-level areas were concentrated in western provinces such as Gansu, Xinjiang, Ningxia, Qinghai, and Inner Mongolia, and less developed central provinces such as Heilongjiang and Jilin. Low-level areas of tourism economic development areas were scattered in the eastern, central, and western provinces represented by Fujian, Jiangxi, and Shaanxi, respectively. Medium-level areas of tourism economic development were scattered in the eastern, central and western provinces represented by Hebei, Anhui, and Sichuan, respectively. Tourism economic development in Tianjin, Liaoning, Henan, and Shanghai were at high-level areas. In 2013, the higher-level and lower-level areas of tourism economic development were continuously and stably distributed in the eastern and northwestern regions. Low-level areas were concentrated in southern China, represented by Hunan and Guangxi. Medium-level areas of tourism economic development were distributed in provinces such as Fujian, Hebei, and Shaanxi. Provinces such as Sichuan, Hubei, Henan, Anhui, Shanghai, and Tianjin had a high-level area of tourism economic development. In 2018, the higher-level areas of tourism economic development were maintained in the eastern coastal areas, while the lower-level areas of tourism economic development were basically concentrated in the northwest. Tourism economic development in western provinces such as Sichuan, Yunnan, and Guangxi jumped to a high-level area. Tourism economic development in provinces such as Hunan and Henan was located at a mediumlevel area. However, tourism economic development in provinces such as Fujian, Shanxi and Guizhou was located at a low-level area. In addition, the number of provinces in China whose tourism economic development index reached the medium (and above) level increased from 16 in 2009 and 2013 to 18 in 2018, and the overall development was above the medium level. But the number of provinces at the lower-level area as a proportion of the total study sample size showed an increasing trend over time, indicating that the imbalance of China's tourism economic development level is prominent. 


\subsection{Characteristics of Ecological Environment Development}

The eco-environmental stress index was calculated by Equation (5) from 2009 to 2018, and the results are shown in Figure 4. It was also integrated with ArcGIS 10.2 to plot the evolution trend of the spatial pattern of China's eco-environmental stress (Figure 5).

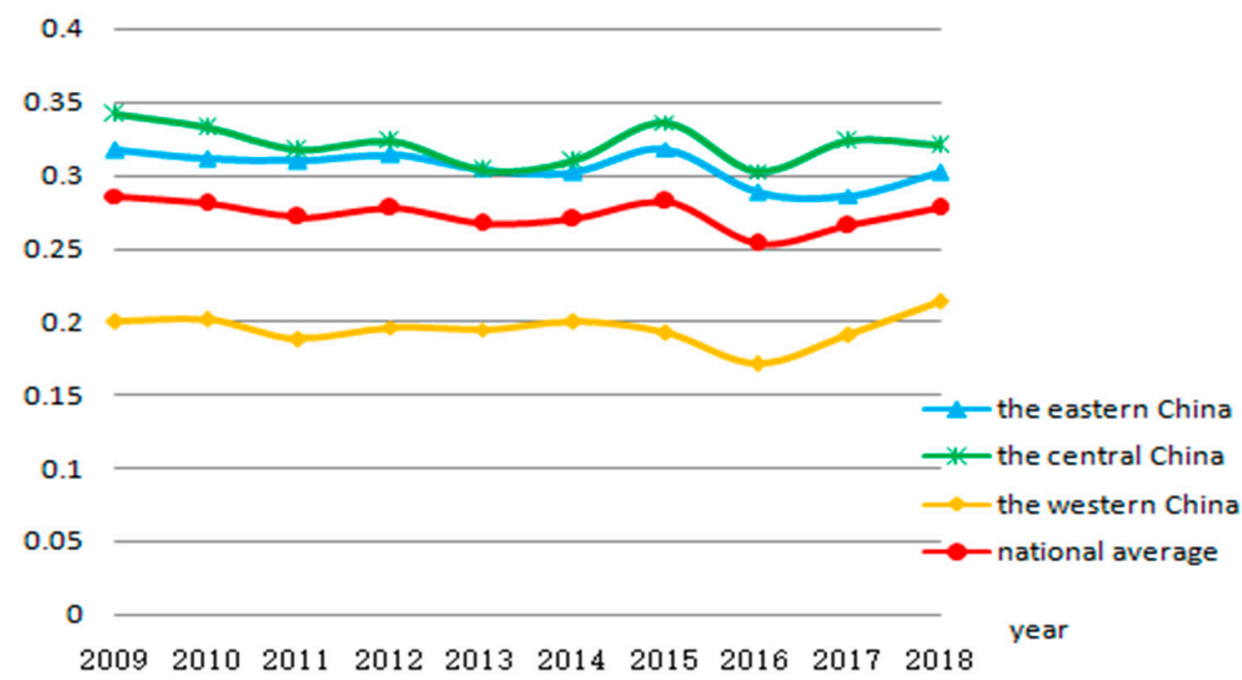

Figure 4. Temporal variation trend of eco-environmental stress index from 2009 to 2018 in China.

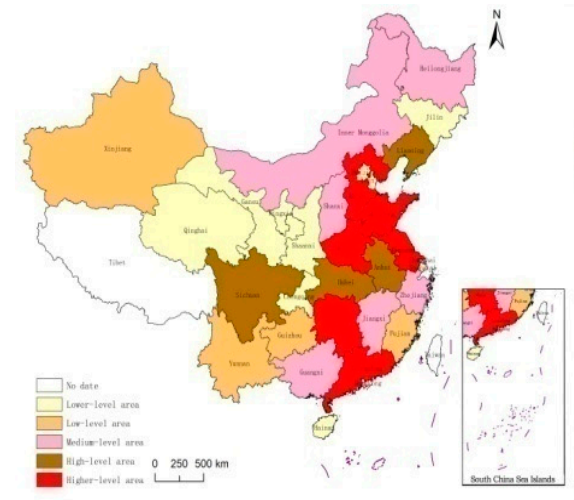

(a) 2009

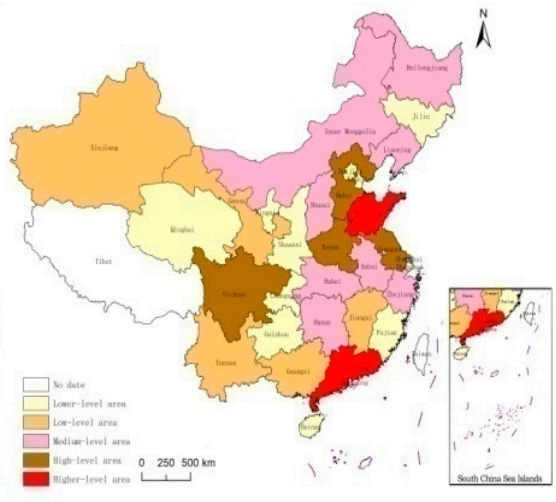

(b) 2013

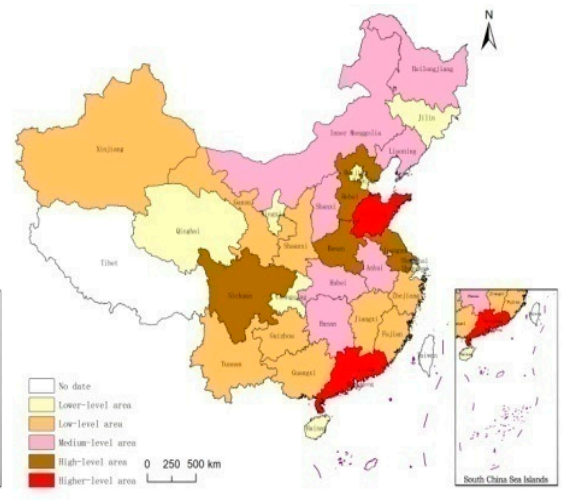

(c) 2018

Figure 5. Spatial pattern of eco-environmental stress index from 2009 to 2018 in China. (a-c) year pattern of ecoenvironmental stress index.

According to the perspective of temporal changes, China's eco-environmental stress as a whole presents the characteristics of fluctuating increase. The eco-environmental stress index of Eastern, Central, and Western China has significant differences, which show fluctuating changes over time. Among them, the eco-environmental stress index of the central region is greater than that of the eastern and western regions, and the eco-environmental stress index of both the central and eastern regions is higher than the national average ecological environment stress. However, the eco-environmental stress of the western region has been in a low value state, lower than the national average stress on the ecological environment. The trough value of China's eco-environmental stress index appeared in 2011, 2013, and 2016. ButChina's eco-environmental stress index reached its peak in 2015. After 2016, China's eco-environmental stress index gradually increased, reflecting the increasing trend of China's eco-environmental stress, and the increasing consumption of resources, environmental pollution, and the degree of threat to nature. 
In terms of spatial evolution pattern, eco-environmental stress index can be divided into five stages. The result shows an overall trend of "high in the east and low in the west, high in the south and low in the north". In 2009, higher-level areas of eco-environmental stress were concentrated in central regions such as Henan, and eastern coastal regions such as Jiangsu and Shandong. High-level areas were scattered in provinces such as Sichuan, Hubei, Anhui, and Liaoning, while medium-level areas were concentrated in six provinces such as Guangxi, Shaanxi, and Inner Mongolia. However, the number of lower-level and low-level areas reached 13, mainly in the western region. In 2013, Guangdong and Shandong were the only provinces with higher-level of eco-environmental stress, and the number of high-level areas increased by 1 compared to 2009. The eco-environmental stress levels of Sichuan and Henan have been maintained at a high-level area, and the number of medium-level areas increased by 2 compared to 2009. In addition, the eco-environmental stress in provinces such as Liaoning, Anhui, and Hubei decreased, the number of lowerlevel and low-level areas increased to 15. And the eco-environmental stress in provinces such as Jiangsu and Fujian weakened. In 2018, the eco-environmental stress index of provinces with high and higher level-areas remained unchanged compared to 2009, and the number of provinces in the medium-level area decreased by one. Mainly, the reason is that the eco-environmental stress in Zhejiang was reduced to a lower-level status. With the exception of Shaanxi, Guizhou, and Fujian, where the eco-environmental stress level has risen from a low-level to a lower-level state, the eco-environmental stress values of provinces that were previously at a low-level and lower-level state remained unchanged. On the whole, China's eco-environmental stress has decreased over time, but has remained at a higher-level in the central region and some eastern provinces, and is obviously weaker in the western region.

\subsection{Evaluating the Decoupling State between Tourism Economic Development and Ecological Environmental Stress}

To more comprehensively reflect the phased changes of the relationship between tourism economic development and eco-environmental stress, based on the principle of equidistant sampling, the study period (2009 to 2018) was divided into two periods: 20092013 (T1) and 2014-2018 (T2). It will carry out comparative analysis of decoupling state, and the specific results are shown in Table 2. According to the results, the research shows that the number of decoupling types of tourism economic development and eco-environmental stress in China presents a diversified development trend with different development stages.

During the T1 period, the decoupling types included types $1-8$, and 16 provinces in a decoupling state (including strong decoupling, weak decoupling, and recessive decoupling) comprised Hebei, Chongqing, and Inner Mongolia, accounting for $53.33 \%$ of the total sample size, of which strong decoupling accounted for $40 \%$. Four provinces in a coupling state (including recessive coupling and expansive coupling) comprised Jiangsu, Henan, Hainan, and Ningxia, accounting for 13.34 of the total sample size. Ten provinces in a negative decoupling state (including expansive negative decoupling, strong negative decoupling, and weak negative decoupling) comprised Beijing, Heilongjiang, and Gansu, accounting for $33.33 \%$ of the total sample size. The results reveal that the T1 period is mainly dominated by decoupling state, during which the development of China's tourism industry is relatively low. The development of tourism economy exerted less stress on the ecological environment. 
Table 2. Decoupling degree of China's tourism economic development and ecological environment stress.

\begin{tabular}{|c|c|c|c|c|c|c|c|c|}
\hline \multirow{2}{*}{ Province/Indicator } & \multicolumn{4}{|c|}{ T1 (2009-2013) } & \multicolumn{4}{|c|}{ T2 (2014-2018) } \\
\hline & $\triangle$ TFDI & $\triangle \mathrm{EEPI}$ & DI & Decoupling Status & $\triangle$ TFDI & $\triangle$ EEPI & DI & Decoupling Status \\
\hline Beijing & -0.1131 & -0.0504 & 0.4456 & Weak negative decoupling & -0.0771 & -0.1246 & 1.6166 & Recessive decoupling \\
\hline Tianjin & -0.1432 & 0.0347 & -0.2424 & Strong negative decoupling & 0.1773 & -0.1140 & -0.6431 & Strong decoupling \\
\hline Hebei & 0.0055 & -0.0293 & -5.3357 & Strong decoupling & 0.1698 & -0.0663 & -0.3903 & Strong decoupling \\
\hline Shanxi & -0.0137 & -0.0300 & 2.1887 & Recessive decoupling & 0.4155 & 0.0581 & 0.1399 & Weak decoupling \\
\hline Inner Mongolia & 0.1321 & -0.0434 & -0.3286 & Strong decoupling & 0.1950 & 0.2184 & 1.1196 & Expansive coupling \\
\hline Liaoning & -0.1634 & -0.1004 & 0.6146 & Weak negative decoupling & -0.2591 & 0.0647 & -0.2496 & Strong negative decoupling \\
\hline Jilin & 0.1276 & -0.0785 & -0.6155 & Strong decoupling & 0.3042 & 0.0301 & 0.0991 & Weak decoupling \\
\hline Heilongjiang & -0.0762 & 0.1769 & -2.3221 & Strong negative decoupling & -0.0152 & 0.2125 & -14.0097 & Strong negative decoupling \\
\hline Shanghai & -0.1267 & -0.0857 & 0.6767 & Weak negative decoupling & 0.0358 & -0.2346 & -6.5556 & Strong decoupling \\
\hline Jiangsu & -0.0390 & -0.0361 & 0.9243 & Recessive coupling & -0.0355 & 0.0442 & -1.2466 & Strong negative decoupling \\
\hline Zhejiang & -0.0202 & 0.0321 & -1.5887 & Strong negative decoupling & -0.0649 & -0.1069 & 1.6465 & Recessive decoupling \\
\hline Anhui & 0.3265 & -0.1182 & -0.3621 & Strong decoupling & 0.0445 & 0.0841 & 1.8904 & Expansive negative decoupling \\
\hline Fujian & 0.1074 & -0.0744 & -0.6923 & Strong decoupling & 0.0887 & -0.0374 & -0.4216 & Strong decoupling \\
\hline Jiangxi & 0.0294 & -0.1449 & -4.9340 & Strong decoupling & 0.3466 & 0.0686 & 0.1981 & Weak decoupling \\
\hline Shandong & 0.0316 & -0.0741 & -2.3456 & Strong decoupling & 0.0101 & 0.1622 & 16.0458 & Expansive negative decoupling \\
\hline Henan & -0.1366 & -0.1210 & 0.8856 & Recessive coupling & 0.3490 & 0.0283 & 0.0810 & Weak decoupling \\
\hline Hubei & 0.1725 & -0.1966 & -1.1400 & Strong decoupling & -0.0031 & 0.0026 & -0.8577 & Strong negative decoupling \\
\hline Guangdong & 0.0443 & 0.0134 & 0.3034 & Weak decoupling & 0.0086 & 0.0424 & 4.8985 & Expansive negative decoupling \\
\hline Guangxi & 0.1133 & -0.2970 & -2.6210 & Strong decoupling & 0.5212 & -0.2327 & -0.4465 & Strong decoupling \\
\hline Hainan & -0.3436 & -0.3488 & 1.0151 & Recessive coupling & 0.9068 & -0.5931 & -0.6541 & Strong decoupling \\
\hline Chongqing & 0.0284 & -0.2298 & -8.1045 & Strong decoupling & -0.1930 & -0.0338 & 0.1753 & Weak negative decoupling \\
\hline Sichuan & 0.1866 & -0.0045 & -0.0241 & Strong decoupling & 0.2561 & 0.2501 & 0.9766 & Expansive coupling \\
\hline Guizhou & -0.0979 & -0.2458 & 2.5104 & Recessive decoupling & 0.2999 & 0.1043 & 0.3478 & Weak decoupling \\
\hline Yunnan & -0.0234 & -0.0083 & 0.3531 & Weak negative decoupling & 0.3706 & -0.0943 & -0.2544 & Strong decoupling \\
\hline Shaanxi & 0.2793 & -0.0045 & -0.0160 & Strong decoupling & 0.2511 & 0.0199 & 0.0794 & Weak decoupling \\
\hline Gansu & 0.0900 & 0.4069 & 4.5212 & Expansive negative decoupling & 0.3321 & 0.3646 & 1.0979 & Expansive coupling \\
\hline Qinghai & -0.2097 & 0.5800 & -2.7662 & Strong negative decoupling & 0.4577 & 0.3654 & 0.7980 & Weak decoupling \\
\hline Ningxia & 0.1810 & 0.1636 & 0.9037 & Expansive coupling & 3.6645 & 0.2173 & 0.0593 & Weak decoupling \\
\hline Xinjiang & 0.0520 & 0.2313 & 4.4473 & Expansive negative decoupling & 0.1884 & 0.0748 & 0.3970 & Weak decoupling \\
\hline
\end{tabular}


During the T2 period, there were 7 types of decoupling (except for the recessive coupling type 4), and the number of provinces in decoupling state reached 18, accounting for $60 \%$ of the total number of samples, of which strong decoupling accounted for $23.33 \%$. The number of provinces in negative decoupling state was 9 , accounting for $30 \%$ of the total number of samples. Only three provinces, including Inner Mongolia, Sichuan, and Gansu were in a state of expansive coupling, accounting for $10 \%$. It can be seen that the number of strong decoupling in the optimal state in the T2 period decreased, and the number of provinces in the coupling state also decreased. While the difference between the number of provinces in the decoupling state and the number of provinces in the negatively decoupling state became larger. It leads to promote the extreme phenomenon of the stress of tourism economic development on the ecological environment. In summary, the decoupling relationship between China's tourism economic development and ecological environment shows fluctuating and changing dynamics, presenting alternating changes in two of the three: Decoupling, coupling, and negative decoupling. The repeated changes in the decoupling state of China's tourism economic development and ecological environment, and the imbalance of the decoupling relationship among provinces more intuitively indicate that: The relationship between China's tourism economic development and ecological environment is in an unstable and unsustainable state.

In this study, ArcGIS 10.2 was used to explore the spatial heterogeneity of decoupling states in T1 and T2 periods, and the spatial pattern evolution map was drawn (Figure 6). The Moran's I value was estimated to be 0.1024 and 0.1517 in T1 and T2 periods. Respectively, it indicates that there is weak spatial autocorrelation between tourism economic development and eco-environmental stress in the two development stages, and it shows an increasing trend of change. In addition, the Z-value and $P$-value corresponding to Moran's I in the T1 period were 1.6372 and 0.1016 . While the Z-value and $P$-value corresponding to Moran's I in the T2 period were 2.2403 and 0.0251 . The $Z$-value of two periods are greater than 1.96 only in the T2 period, and pass the significance test at the 0.05 level. In a word, during the study period, the decoupling relationship between China's tourism economic development and eco-environmental stress has a certain positive spatial correlation. The decoupling state of tourism economic development and eco-environmental stress among provinces presents certain agglomeration characteristics, but the degree of spatial agglomeration is not high.

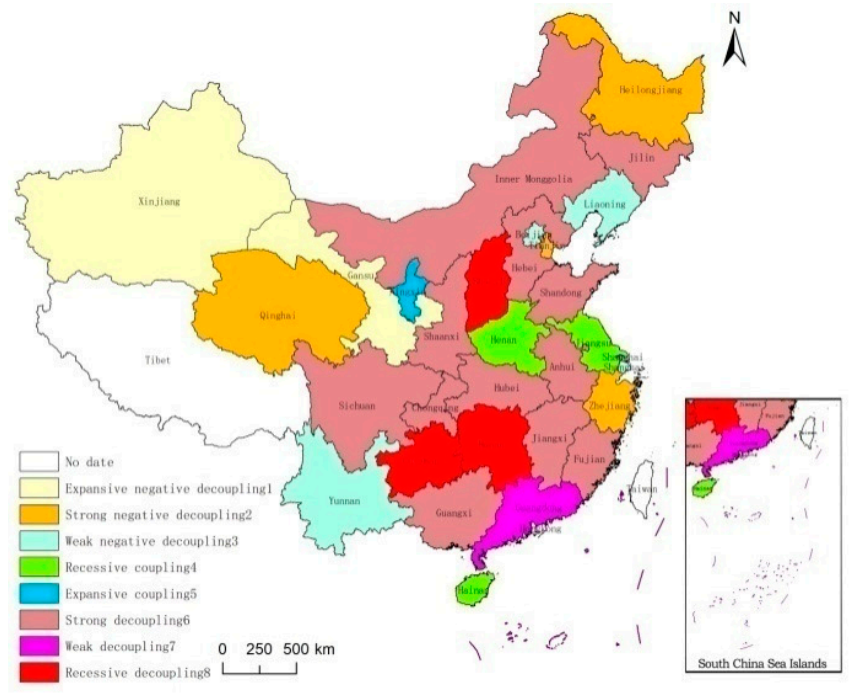

(a) 2009-2013

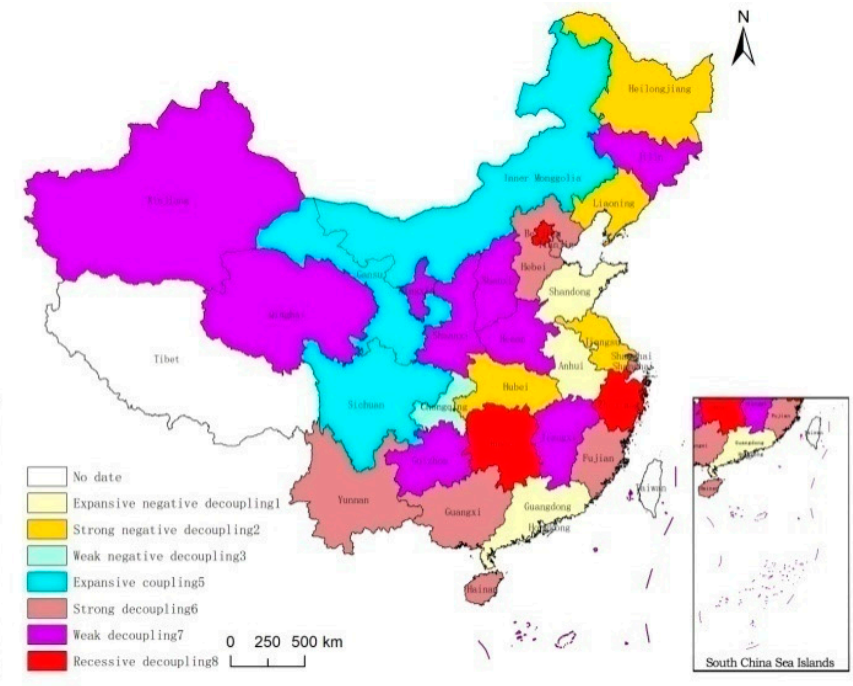

(b) 2014-2018

Figure 6. The evolution of the spatial pattern of the decoupling state of tourism economic development and ecological environmental stress in China. $(\mathbf{a}, \mathbf{b})$ decoupling state of tourism economic development and ecological environmental. 
In Figure 6,various decoupling types are scattered in different provinces of China, and the decoupling relationship between tourism economic development and eco-environmental stress in the eastern, central and western regions of China presents different patterns of change with different development periods. The number of provinces with very good status of strong decoupling type 6 changed from 12 in the T1 period to 7 in the T2 period. The ratio of the number distributed in the eastern, central, and western provinces changed from 4:3:5 in the T1 period to 5:0:2 in the T2 period, respectively, with a tendency for the spatially good status of decoupling relationships to weaken. The number of weak decoupling type 7 and recessive decoupling type 8 increased from 4 in the T1 period to 12 in the T2 period. And the ratio of the number distributed in the eastern, central and western provinces changed from 1:2:1 in T1 to T2 of 2:5:5. It reflects that the stress on the ecological environment generated by tourism economic development in the central and western regions tended to decrease. The ratio of the number of provinces with recessive coupling type 4 and expansive coupling type 5 changed from 3:1 in the T1 period to 0:3 in the T2 period, with the coupling state of the decoupling relationship tending to be good. The ratio of the number of provinces in the poor decoupling state of expansive negative decoupling type 1 , strong negative decoupling type 2 , and weak negative decoupling type 3 changed from 2:4:3 in T1 to 3:4:1 in T2. And the number of provinces in the negative decoupling relationship distributed in the eastern, central and western parts of China changed from 4:1:4 in T1 to 5:2:1 in T2. Thisindicates that the stress of tourism economic development on the ecological environment in the eastern and central parts of China tends to increase. Whereas the stress on the ecological environment in the western part of China tends to weaken.

In summary, the strong decoupling relationship between China's tourism economic development and the optimization of ecological environment tends to weaken. In the T1 period, the decoupling status of the western region is better than that of the eastern and central regions, but the overall decoupling degree of the eastern region in the T2 period is better than that of the central and western regions. Meanwhile, the negative decoupling status of some provinces in the eastern region should not be underestimated. In recent years, the country has vigorously implemented supply-side structural reform measures, optimized and adjusted for an industrial structure, developed for the tourism industry. Meanwhile, the country is focusing on the quality and efficiency of tourism and the overall implementation of the concept of green development, which reduces the stress on the ecological environment to a certain extent. Finally, the decoupling relationship between tourism economic development and ecological environment tends to be optimized. Therefore, we should gradually reduce the stress on ecological environment in the central region, enhance the level of tourism economic development in the western region, actively play a radiation-driven role in the eastern region, and comprehensively promote the coordinated development between regions. So it is more conducive to the overall decoupling and optimization of China's tourism economic development and ecological environment stress.

\section{Analysis for the Reasons of the Change of Decoupling Relationship}

This study takes the decoupling status of Chinese provinces in the T2 (2014-2018) period as the benchmark. Based on the results of the three types of decoupling status, such as decoupling, negative decoupling, and coupling, each indicator of tourism enterprise scale (TS), tourism capital investment (TI), tourism reception level (TR), tourism industry performance $(\mathrm{TP})$, resource consumption condition (RC),environmental pollution status (EP), and nature threat level (NT) in Table 1 were selected. They were wanted to analyze and dominate the intrinsic reasons that dominate the changes in the interrelationship between tourism economic development and ecological environmental stress in China. 


\subsection{Decoupling Status of Provincial Domains}

\subsubsection{Regression Model Construction}

In the decoupling state, the resource consumption index, environmental pollution status index and natural threat index were fitted with the indicators of tourism economic development respectively by the least square method. The regression analysis was carried out using the fixed-effect model by combining the test results, thus the fitted equation models were obtained as follows:

$$
\begin{gathered}
\mathrm{RC}_{\mathrm{ij}}=0.0389+0.5325 \mathrm{TS}_{\mathrm{ij}}-0.2916 \mathrm{TI}_{\mathrm{ij}}+1.037 \mathrm{TR}_{\mathrm{ij}}-0.5227 \mathrm{TP}_{\mathrm{ij}}(\mathrm{i}=1,2, \ldots 19 ; \mathrm{j}=1,2, \ldots 5) \\
\mathrm{EP}_{\mathrm{ij}}=0.0533+0.5168 \mathrm{TS}_{\mathrm{ij}}-0.9038 \mathrm{TI}_{\mathrm{ij}}+1.7974 \mathrm{TR}_{\mathrm{ij}}-0.5218 \mathrm{TP}_{\mathrm{ij}}(\mathrm{i}=1,2, \ldots 19 ; \mathrm{j}=1,2, \ldots 5) \\
\mathrm{NT}_{\mathrm{ij}}=1.2182 \mathrm{TR}_{\mathrm{ij}}-0.3116 \mathrm{TP}_{\mathrm{ij}}(\mathrm{i}=1,2, \ldots 19 ; \mathrm{j}=1,2, \ldots 5) .
\end{gathered}
$$

According to the fitting results, the fitting degree of resource consumption index, environmental pollution status index and the indicators of tourism economic development is general. The corresponding determination coefficient $R^{2}$ are $0.6007,0.5165$, respectively, and the $P$ value are both 0.0000 , which is obviously significant. Resource consumption index and environmental pollution are significantly positively correlated with tourism enterprise size (TS) and tourism reception level (TR), and negative correlations with tourism capital investment (TI) and tourism industry performance (TP) (Table 3). This indicates that as the scale of tourism enterprises increases and the level of tourism reception increases, the degree of resource consumption appears to increase significantly. However, with the increase of tourism capital investment and tourism industry performance, the degree of resource consumption decreases instead. The improvement of tourism enterprise scale and tourism reception level, the degree of environmental pollution increases.

Table 3. Regression results of the decoupling state of provincial eco-environmental stress and various tourism economic development indicators. Abbreviations: Tourism enterprise scale (TS), tourism capital investment (TI), tourism reception level (TR), tourism industry performance (TP), constant term $(\mathrm{C})$.

\begin{tabular}{cccccc}
\hline Index & Variable & Coef. & Std.Err. & t-Stat. & Prob. \\
\hline \multirow{2}{*}{ Resource } & C & 0.0389 & 0.0075 & 5.1583 & $0.0000^{* *}$ \\
consumption & TS & 0.5325 & 0.1018 & 5.2285 & $0.0000^{* *}$ \\
conditions & TI & -0.2916 & 0.1213 & -2.4028 & $0.0183^{*}$ \\
& TR & 1.0370 & 0.1957 & 5.2996 & $0.0000^{* *}$ \\
& TP & -0.5227 & 0.1292 & -4.0466 & $0.0001^{* *}$ \\
\hline Environmental & C & 0.0533 & 0.0119 & 4.4657 & $0.0000^{* *}$ \\
pollution status & TS & 0.5168 & 0.1610 & 3.2089 & $0.0018^{* *}$ \\
& TI & -0.9038 & 0.1919 & -4.7102 & $0.0000^{* *}$ \\
& TR & 1.7974 & 0.3094 & 5.8087 & $0.0000^{* *}$ \\
& TP & -0.5218 & 0.2043 & -2.5542 & $0.0123^{*}$ \\
\hline \multirow{2}{*}{ Natural threat } & C & 0.0184 & 0.0088 & 2.0886 & 0.0396 \\
level & TS & -0.1678 & 0.1189 & -1.4104 & 0.1619 \\
& TI & -0.0990 & 0.1417 & -0.6985 & 0.4866 \\
& TR & 1.2182 & 0.2285 & 5.3303 & $0.0000^{* *}$ \\
\hline TP & -0.3116 & 0.1509 & -2.0652 & $0.0418^{*}$ \\
\hline
\end{tabular}

Note: ${ }^{* *}$ and ${ }^{*}$ represent passing significance tests at 0.01 and 0.1 levels.

The degree of natural threat and the indicators of tourism economic development have a low degree of fit, with a determination coefficient $\mathrm{R}^{2}$ of 0.3085 and a $P$-value of 0.0000 . The degree of natural threat showed a positive correlation with tourism reception level (TR) and a negative correlation with tourism industry performance (TP). It shows that the continuous increase in tourism reception level and the increasing number of tourists, the degree of natural threat emerges continuously. 


\subsubsection{Cause Analysis}

Innovation-driven green transformation is the key to achieving harmony between tourism economic development and ecological environment. The tourism economic development are decoupled from the eco-environmental stress in Beijing, Tianjin, Shanghai, and Zhejiang. It has a significant positive correlation with the scale of tourism enterprises and the level of tourism reception but presents a negative correlation with tourism capital investment and tourism industry performance. The reason is that in the decoupling state, the provinces pay more attention to the construction of tourism infrastructure and the development of tourism market, continuously optimize and adjust the structure of tourism industry. In addition, they improve the quality of tourism service, make full use of existing resources to implement green innovation, and vigorously promote the green transformation of tourism.It is worth noting that most of the national eco-tourism demonstration zones are concentrated in these provinces. The state vigorously cultivates emerging demonstration zones, strengthens innovation drive, adheres to the path of green and high-quality development, and provides a good market environment for tourism economic development. So, they are playing an important role in the sustainable coordination of tourism economic development and ecological environment.

The ecological environment has a positive effect on tourism economic development. Western regions such as Guizhou, Yunnan, Qinghai, and Guangxi have better ecological conditions and rich natural resources, and have a number of national natural scenic areas due to their superior natural environmental conditions, so as to provide a guarantee for the growth of the tourism economy. However, due to the relatively backward traffic conditions in the western region and the relative lack of talents and funds, the development of the tourism economy is thus restricted to a certain extent and the level of development is relatively backward, which in turn produces little stress on the ecological environment. The level of tourism economic development and eco-environmental stress increase or decrease simultaneously, which eventually puts the two in a good state of decoupling.

\subsection{Provinces with Negative Decoupling Status}

\subsubsection{Regression Model Construction}

According to the above analysis methods and principles for provinces with decoupling status, regression models of ecological environment stress and various tourism economic development indicators in provinces with negative decoupling status are as follows:

$$
\begin{gathered}
\mathrm{RC}_{\mathrm{ij}}=0.5206 \mathrm{TS}_{\mathrm{ij}}+1.0665 \mathrm{TI}_{\mathrm{ij}}(\mathrm{i}=1,2, \ldots 8 ; \mathrm{j}=1,2, \ldots 5) \\
\mathrm{EP}_{\mathrm{ij}}=0.6791 \mathrm{TS}_{\mathrm{ij}}+0.0528(\mathrm{i}=1,2, \ldots 8 ; \mathrm{j}=1,2, \ldots 5) .
\end{gathered}
$$

The results show that the resource consumption index and environmental pollution status index fit well with the indicators of tourism economic development. Determination coefficient $\mathrm{R}^{2}$ are 0.9002 and 0.7689 , respectively, and the $P$ value are both 0.0000 , both of which have obvious significance. The resource consumption index showed significant positive correlation with tourism enterprise scale (TS) and tourism capital investment (TI) (Table 4), insignificant negative correlation with tourism reception level (TR), and insignificant positive correlation with tourism industry performance (TP).This indicates that with the increasing scale of tourism enterprises and the increase of tourism capital investment, the degree of resource consumption also appears to increase significantly.

Environmental pollution status showed positive correlation with tourism enterprise scale (TS), insignificant positive correlation with tourism capital investment (TI) and tourism industry performance (TP), and insignificant negative correlation with tourism industry performance (TP). It reflects that the increase in tourism enterprise scale leads to a greater degree of environmental pollution.

In addition, The natural threat index has little significance with each index of tourism economic development. There was no significant linear relationship, indicating that tourism 
economic development does not pose a threat to the natural ecological environment or the degree of threat is very weak.

Table 4. Regression results of decoupling negative state of provincial ecological environmental stress and various tourism economic development indicators.

\begin{tabular}{cccccc}
\hline Index & Variable & Coef. & Std.Err. & t-Stat. & Prob. \\
\hline \multirow{2}{*}{ Resource } & C & 0.0177 & 0.0190 & 0.9325 & 0.3574 \\
consumption & TS & 0.5206 & 0.1417 & 3.6747 & $0.0008^{* *}$ \\
conditions & TI & 1.0665 & 0.3128 & 3.4090 & $0.0017^{* *}$ \\
& TR & -0.4310 & 0.3428 & -1.2572 & 0.2170 \\
& TP & 0.3780 & 0.2662 & 1.4199 & 0.1645 \\
Environmental & C & 0.0528 & 0.0224 & 2.3631 & $0.0238^{*}$ \\
pollution status & TS & 0.6791 & 0.1671 & 4.0635 & $0.0003^{* *}$ \\
& TI & 0.3207 & 0.3690 & 0.8690 & 0.3908 \\
& TR & -0.0243 & 0.4044 & -0.0602 & 0.9524 \\
& TP & 0.1269 & 0.3141 & 0.4041 & 0.6886 \\
\hline
\end{tabular}

Note: ${ }^{* *}$ and ${ }^{*}$ represent passing significance tests at 0.01 and 0.1 levels.

\subsubsection{Cause Analysis}

Tourism capital investment, as the leading driving factor, is unsustainable. The resource consumption index of developed coastal provinces such as Liaoning, Jiangsu, Shandong, and Guangdong, as well as important provinces such as Heilongjiang, Anhui, Hubei, and Chongqing, show a significant positive correlation with tourism enterprise size (TS) and tourism capital investment (TI). The reason for this is that developed provinces such as Shandong and Jiangsu rely on the existing geographical advantageous conditions, convenient tourism transportation, rich tourism resource endowment, and strong supporting industrial support to attract a large number of the population gathering. It results in the increasing consumption of resources and the increasing stress of tourism economic development on the ecological environment. In addition, many tourism investors prefer to choose Liaoning and other coastal provinces with superior conditions, so as to expand the scale of tourism development. At one time, the pursuit of high economic benefits will also consume a lot of resources, and this investment-driven tourism economic development is unsustainable and will only continue to increase the stress on the ecological environment.

There is a significant dependence on a resource-driven approach to economic development. In the state of negative decoupling, the province is rich in tourism resources, and the development of tourism economy is mostly in the mature stage. However, the tourism economy in these provinces mostly follows the rough economic development method of resource consumption, relying on the existing rich tourism resources for overexploitation, incomplete transformation of related industries. They lack new supporting industries, insufficient investment in fixed assets and environmental management, and insufficient tourism human capital. It results in adverse social, environmental, and economic effects, and makes the development of the tourism economy still exerts great stress on the ecological environment.

\section{Conclusions and Discussion}

\subsection{Conclusions}

Based on the measurement of China's tourism economic development index and eco-environmental stress index, combined with ArcGIS technology, this paper analyzes spatial and temporal evolution characteristics, evaluate the correlation between tourism economic development and eco-environmental stress with the decoupling model. At the same time, it aims at analyzing the reasons for their changes, resulting in the following conclusions:

The tourism economic development and eco- environmental stress show certain characteristics of space-time effect. During the study period, China's tourism economic 
development and eco-environmental stress index as a whole showed a fluctuating trend of first decreasing and then increasing. The level of tourism economic development kept increasing or decreasing while the eco-environmental stress also exhibited an increasing or decreasing trend. China's tourism economic development and eco-environmental stress show obvious spatial level differences and weak agglomeration characteristics, with prominent regional imbalance. The level of tourism economic development in the eastern region is generally higher than that in the central and western regions, while the ecoenvironmental stress in the central region is greater than that in the eastern and western regions.

The relationship between tourism economic development and ecological environmental stress of China's provinces has experienced eight states, and the optimal strong decoupling relationship tends to weaken. During the study period, most of the provinces showed a trend of alternate changes in two out of three decoupling, coupling, and negative decoupling. Because most of provinces failed to achieve overall decoupling optimization in space under the constraints of local policy orientation and regional economic development level, the decoupling status was always in an unsustainable and non-optimal stage. Each province should start with technological progress, effectively improve the utilization efficiency of tourism resources and the ability of environmental governance, implement low-carbon, coordinated and green tourism development policies, and correctly handle the relationship between tourism economic development and ecological environment. Moreover, they should build a long-term mechanism for the coordinated development of the two, explore the economic development path of ecological tourism in line with the actual situation of each region. In particular, under the current situation of the outbreak of COVID-19 and the normal prevention and control, the tourism industry has been greatly impacted by the COVID-19 pandemic [47], and the tourism economy has been seriously deteriorated. Therefore, it is necessary to accelerate the sustainable recovery of social health, the environment and the tourism economy [48]. Finally, it is important to promote the innovation of China's green tourism development mode; strengthen the development of ecological health tourism to improve immunity and optimize the ecological environment; promote the integration of "health preservation + ecological tourism + health treatment". The purpose is to ensure the tourism economic development and eco-environmental stress towards a benign and sustainable decoupling direction.

The reasons for the differential changes in the decoupling state of tourism economic development and ecological environmental stress in China's provinces come from the investment-driven, resource-driven, innovation-driven, and environmental compliance push. China's tourism economic development is mostly resource-driven and investmentdriven as an important driving force. With the continuous improvement of the scale of tourism enterprises and tourism reception level, the stress of tourism economic development on the ecological environment is uneven, and the instability and unsustainability are obvious. Strengthening innovation drive and paying attention to natural ecology are the key driving forces to get rid of the stress of tourism economic development on ecological environment, so as to ensure the coordinated and symbiotic development of tourism economic development and ecological environment.

\subsection{Discussion}

At present, tourism economic development and ecological environment issues are increasingly favored by the government and the industry. How to coordinate the relationship between tourism economic development and ecological environmental stress has become a major challenge for the regional economy to achieve sustainable development. This study not only attempts to construct a decoupling evaluation model of tourism economic development and ecological environmental stress, but also uses the spatiotemporal evolution method to evaluate the internal law of tourism economic development and ecological environmental stress. Therefore, there is some innovation in the research method. Moreover, this study combines with geographic information systems and the theory of 
the statistical to analysis of tourism economic development and ecological environmental stress of spatial heterogeneity. Exploring the causes of the change in decoupling state by classificationis conducive to exploring different tourism development modes conducive to the sustainable development of ecological environment. So, this study contributes certain innovative ideas. Its finding will help the tourism department and the environmental department better understand the current development situation, provide a reference for the national formulation of macroeconomic control policies and environmental protection policies, and support the realization of high-quality tourism development and the sustainable development of the ecological environment. However, there are limitations to this study. The first limitation concerns the scope of the data analysis. The data used in this study is only for the analysis of China's national conditions, so the results of this study cannot be generalized. In future studies, researchers should analyze the situation of globalization more widely, to obtain more practical and universal results for globalization. The second limitation is that the research scale is limited to the provincial level. Due to the lack of data, future research needs to extend the analysis to the city and county scales to compensate for the missing data and reveal the law of interaction between tourism economic development and ecological environmental stress on a more refined scale. Finally, due to space constraints, we could not discuss spatial analysis on the data for each year, and only selected representative years for analysis.

Because the period covered in this study was from 2009 to 2018, the data analysis did not examine the influence of the COVID-19 pandemic, and it is necessary to conduct further research. In future studies, further research will be carried out on the recovery of tourism economic development and sustainable development of ecological environment under the situation of COVID-19 epidemic. At the same time, the impaction assessment of COVID-19 epidemic on tourism and ecological environment will be discussed, and the internal action rules of the two systems of tourism economic development and ecological environment stress will be analyzed in depth. Future research should seek answer the following questions:

- What is the impact of COVID-19 pandemic on tourism economic development and ecological environment? Did the pandemic bring about a radical change to the tourism economy? Can it impose irreversible harm on the ecological environment?

- How can the tourism economy develop in a sustainable and high-quality way after the COVID-19 pandemic? How can "zero pressure" on the ecological environment be achieved?

- What sustainable development mode can support the harmonious coexistence of tourism with the ecological environment?

Author Contributions: X.Q. had the original idea for the study and wrote the first draft. Relative contribution of the authors is indicated by order of listing. Both authors framed the discussion in accordance with an initial idea X.L. edited the manuscript and provided a critical final review. All authors have read and agreed to the published version of the manuscript.

Funding: This study was jointly supported by the BaGui Scholars Program of Guangxi Zhuang Autonomous Region of China (2019BG07).

Data Availability Statement: Not applicable.

Conflicts of Interest: The authors declare no conflict of interest.

\section{References}

1. Guo, X.Y.; Mu, X.Q.; Ding, Z.S.; Ming, Q.Z.; Lu, B. Coordination effect and dynamic relationship between urban ecological environment and tourism economy: A case study of Qujing. Econ. Geogr. 2020, 40, 231-240.

2. Gu, X.; Hunt, C.A.; Lengieza, M.L.; Niu, L.; Wu, H.; Wang, Y.; Jia, X. Evaluating Residents' Perceptions of Nature-Based Tourism with a Factor-Cluster Approach. Sustainability 2021, 13, 199. [CrossRef]

3. Roman, M.; Niedziółka, A. Spatial Diversity of Tourism in the Countries of the European Union. Sustainability 2020, $12,2713$. [CrossRef]

4. Gössling, S. Global environmental consequences of tourism. Glob. Environ. Chang. 2002, 12, 283-302. [CrossRef] 
5. Ye, W.; Xu, X.; Wang, H.; Wang, H.; Yang, H.; Yang, Z. Quantitative assessment of resources and environmental carrying capacity in the northwest temperate continental climate ecotope of China.Environ. Earth Sci. 2016, 75, 868. [CrossRef]

6. Josef, Z.; Jaroslav, K. The concept of carrying capacity in tourism. Amfiteatru. Econ. 2014, 16, 641-654.

7. Yunhe, W.; Jia, L.; Meiqi, Z. Evaluation of tourism environmental carrying capacity in Diaoshuihu National Forest Park. Int. J. Sustain. Dev. Plan. 2020, 15, 77-97.

8. Wenqi, R.; Yongquan, L.; Shuning, Z.; Liu, C. Evaluation and drive mechanism of tourism ecological security based on the DPSIR-DEA model. Tour. Manag. 2019, 75, 609-625.

9. Zhao, X. Coupling relationship and coordinated development of tourism economy and ecological environment. Res. Financ. Issues. 2014, 238-240.

10. Yang, S.; Qin, Y.; Deng, W.; Ji, M. Comparison of coupling relationship between tourism economy and ecological environment in China's top ten urban agglomerations. Stat. Decis. Mak. 2017, 11, 131-134.

11. Cheng, H.; Xu, Q.; Guo, Y. Temporal and spatial evolution of the coupling coordinated development between tourism resources development and ecological environment in China. Econ. Geogr. 2019, 39, 233-240.

12. Weng, G.; Pan, Y.; Li, L. Tourism ecological security level measurement and spatial-temporal evolution analysis based on improved DPSIR-DS model: A case study of five provinces along the Silk Road. Tour. Sci. 2018, 32, 17-32.

13. Liu, J.; Yu, S.; Wang, J. Evaluation and quantitative measurement of coastal tourism environmental carrying capacity: A case study of Shandong Peninsula Blue Economic Zone. China Popul. Resour. Environ. 2012, 22, 163-170.

14. Su, M. Sustainable tourism and community development in tourist destinations. Tour. Tribu. 2014, 29, 8-9.

15. Song, R. Sustainable tourism development under the background of ecological civilization system construction. Ecolog. Econ. 2018, 34, 134-138.

16. Anna, T.; Francesc, L.P. The ISOST index: A tool for studying sustainable tourism. J. Destin. Mark. Manag. 2018, 8, $281-289$.

17. Asmelash, A.G.; Kumar, S. Assessing progress of tourism sustainability:Developing and validating sustainability indicators. Tour. Manag. 2019, 7, 67-83. [CrossRef]

18. Li, X.S.; Qu, F.T.; Guo, Z.X.; Jiang, D.M.; Pan, Y.Q.; Chen, X.L. Decoupling between urban and rural construction land. China Popul. Resour. Environ. 2008, 18, 179-184.

19. Carter, A.P. The economics of technological change. Sci. Am. 1966, 214, 25-31. [CrossRef]

20. OECD. Indicators to Measure Decoupling of Environmental Stress from Economic Growth; OECD: Paris, France, 2002.

21. OECD. Effects of Quantitative Constraints on the Degree of Decoupling of Crop Support Measures; OECD: Paris, France, 2005.

22. Ayres, R.U.; Ayres, L.W.; Warr, B. Energy, power and work in the US economy. Energy 2003, 28, 219-273. [CrossRef]

23. Tapio, P. Towards a theory of decoupling: Degrees of decoupling in the EU and the ease of road traffic in Finland between 1970 and 2001. J. Transp. Policy 2005, 12, 137-151. [CrossRef]

24. Heinz, S.; Steve, H.D.; Thomas, W.; Arne, G.; Yiyong, C.; James, W.; David, N.; Tim, B.; Manfred, L.; Anne, O. Decoupling global environmental stress and economic growth: Scenarios for energy use, materials use and carbon emissions. J. Clean. Prod. 2016, 13, 45-56.

25. Taewook, H. Comparative and relational trajectory of economic growth and greenhouse gas emission: Coupled or Decoupled? Energies 2020, 13, 1-13.

26. Gai, M.; Hu, H.; Ke, L. Decoupling analysis of resources, environment and economic growth in Yangtze River Delta. J. Nat. Resour. 2013, 28, 185-198.

27. Bai, C.; Huang, Y.; Song, W.; Feng, Y. Study on the decoupling of industrial economic development and environmental pollution in the Yangtze River Delta. Environ. Sci. Technol. 2015, 38, 157-163.

28. Wang, Z.; Lu, X.; Han, Z.; Dong, X. The decoupling analysis and rebound effect of China's marine economic growth and resource consumption. Resour. Sci. 2017, 39, 1658-1669.

29. Ding, B.; Yang, S.; Zhao, Y.; Yi, S. Study on the temporal and spatial characteristics and decoupling effects of carbon emissions in the utilization of cultivated land resources in China. China Land Sci. 2019, 33, 45-54.

30. Wang, X.; Shen, D.; Li, W. Decoupling mechanism, model and application of water resources utilization and economic growth. China Popul. Resour. Environ. 2019, 29, 139-147.

31. Wang, X.; Wang, H.; Wang, X. Spatial temporal difference analysis of decoupling relationship between new urbanization level and eco-environmental stress: A case study of Mianyang City, Sichuan Province. China Agric. Resour. Reg. Plan. 2019, 40, 121-129.

32. Zhao, X.; Wang, J.; Guo, B.; Zhai, T.; Liu, J. Expansion, utilization efficiency and decoupling of construction land in coastal areas: A case study of Dafeng District, Jiangsu Province. Soil Water Conserv. Res. 2020, 27, 340-348.

33. Lu, L.; He, J.; Zeng, G. Study on decoupling of economic growth and industrial wastewater discharge from an innovation-driven perspective: A case study of Yangtze River Delta urban agglomeration. Reg. Res. Dev. 2020, 39, 156-162.

34. Fang, Y.L.; Huang, Z.F.; Duan, Z.X.; Wang, K. Coupling and coordinating about tourism development and environment. Econ. Geogr. 2013, 33, 195-201.

35. Guo, X.; Li, Y. Spatial differences and evolutionary characteristics of the level of coordinated development of tourism economy and ecological environment in China. China Popul. Resour. Environ. 2014, 24, 356-359.

36. Zhou, B.; Zhao, K.; Zhong, L.; Chen, T.; Yu, H. Evaluation of the coordinated development of ecosystem health and tourism economy in Zhoushan Islands. Acta Ecol. 2015, 35, 3437-3446. 
37. Xu, F.; Zhao, S.; Du, T.; Zhan, W.; Zhao, Z.; Hao, J.; Zhang, Y. Quantitative evaluation of the stress of regional economic development on ecological environment. China Popul. Resour. Environ. 2004, 8, 32-38.

38. Zhu, J.; Wang, H.; Diao, S.; Gu, Q. Evaluation of ecological environment stress in Northern Shaanxi based on improved correspondence analysis-ES model. J. Beijing Normal Univ. 2016, 52, 466-471.

39. Du, J.; Liu, X.; Hu, Y.; Zhu, S. Evaluation of the decoupling of economic growth and forest ecological environment stress from the perspective of ecological civilization: A comparative study based on the first batch of national ecological civilization experimental areas. J. For. Econ. 2019, 41, 98-107.

40. Rong, X.; Hu, Z.; Xiong, X. Inter-regional differences in the coupling degree of tourism development and ecological civilization construction. Econ. Geogr. 2016, 36, 189-194.

41. Liu, H.; Yang, Q.S.; Zhang, Y. Decoupling analysis of urbanization and ecological environment in Northeast China. Geogr. Sci. 2016, 36, 1860-1869.

42. Huang, M.; Yue, W.; He, X. Decoupling relationship between urban expansion and economic growth and its spatial heterogeneity in the Yangtze Economic Belt. J. Nat. Resour. 2018, 33, 219-232.

43. Tasbasi, A. A threefold empirical analysis of the relationship between regional income inequality and water equity using Tapio decoupling model, WPAT equation, and the local dissimilarity index: Evidence from Bulgaria. Environ. Sci. Pollut. Res. 2020, 9 , 1-14. [CrossRef]

44. Liu, H. Decoupling of China's economic growth and energy consumption: A Study on temporal and spatial differentiation in eastern China. China Popul. Resour. Environ. 2016, 26, 157-163.

45. Ma, R.H.; Pu, Y.X.; Ma, X.D. GIS Spatial Association Pattern Discovery; Science Press: Beijing, China, 2007 ; pp. $103-107$.

46. Kyriakidis, P.; Journel, A.G. Geostatistics models: A review. Math. Geol. 1999, 31, 615-684. [CrossRef]

47. Kitamura, Y.; Karkour, S.; Ichisugi, Y.; Itsubo, N. Evaluation of the Economic, Environmental, and Social Impacts of the COVID-19 Pandemic on the Japanese Tourism Industry. Sustainability 2020, 12, 10302. [CrossRef]

48. Roman, M.; Niedziółka, A.; Krasnodębski, A. Respondents' Involvement in Tourist Activities at the Time of the COVID-19 Pandemic. Sustainability 2020, 12, 9610. [CrossRef] 